\title{
GRAY CURVATURE IDENTITIES FOR ALMOST CONTACT METRIC MANIFOLDS
}

\author{
Raluca Mocanu and Marian Ioan Munteanu
}

\begin{abstract}
Alfred Gray introduced in [8] three curvature identities for the class of almost Hermitian manifolds. Using the warped product construction and the Boothby-Wang fibration we will give an equivalent of these identities for the class of almost contact metric manifolds.
\end{abstract}

\section{Introduction}

In [8], A. Gray introduced three curvature identities, $K_{i}, i=1,2,3$, for almost Hermitian manifolds. These identities have important applications in geometry and topology (for example, some other geometric objects can be constructed on manifolds satisfying $K_{i}$, see e.g. $\left.[12,13]\right)$.

A naturally arising question would be: which are the equivalent identities for the almost contact manifolds?

Concerning this, A. Bonome, L. M. Hervella, and I. Rozas defined in [4] $K_{i \varphi}$-curvature identities $(i=1,2,3)$ for an almost contact metric manifold $(M, \varphi, \xi, \eta, g)$ by using the usual Hermitian structure on $M \times \mathbb{R}$ (the product manifold). Gray proved in [8] that Kaehlerian manifolds satisfy $K_{i}, i=1,2,3$ (curvature identities for almost Hermitian manifolds). In the same spirit, in [4] it is shown that cosymplectic manifolds satisfy $K_{i \varphi}$-identities.

It is known that both cosymplectic and Sasakian manifolds are natural odddimensional versions of Kaehlerian manifolds. We ask what happens if the analogy with the Kaehlerian manifolds is extended to Sasakian manifolds? We bear in mind the fact that a Riemannian manifold $(M, g)$ is Sasakian if the holonomy group of the metric cone on $M:\left(C(M)=\mathbb{R}_{+} \times M, \widetilde{g}=d t^{2}+t^{2} g\right)$

Received July 15, 2008; Revised December 7, 2008.

2000 Mathematics Subject Classification. 53C15, 53C25, 53C55, 53B35, 53D15.

Key words and phrases. almost Hermitian manifolds, almost contact metric manifolds, curvature identities, Boothby-Wang fibration, cone metric, cosymplectic manifolds, Sasakian manifolds, generalized Heisenberg group.

The first author was supported by Grant CEEX ET n. 5883/2006-2008 ANCS Romania.

The second author was supported by Grant PN II ID 398/2007-2010 ANCS Romania. 
reduces to a subgroup of $U\left(\frac{m+1}{2}\right)$, i.e., $(C(M), \widetilde{g})$ is Kaehlerian. (Here $m=$ $\operatorname{dim} M$.)

Inspired by this definition and by [4], we will take a different approach to Gray curvature identities for almost contact metric manifolds. For this purpose we will use the warped product and the Boothby-Wang fibration, two different constructions which lead us to the same result.

\subsection{Gray curvature identities}

An almost Hermitian manifold $(M, J, g)$ is said to satisfy Gray curvature identities $(K 1),(K 2)$ and respectively $(K 3)$, if its Riemann Christoffel curvature tensor satisfies

(K1) $R(X, Y, Z, W)=R(X, Y, J Z, J W)$,

(K2) $R(X, Y, Z, W)=R(J X, J Y, Z, W)+R(J X, Y, J Z, W)+R(J X, Y, Z, J W)$,

(K3) $R(X, Y, Z, W)=R(J X, J Y, J Z, J W)$

for all vector fields $X, Y, Z, W$ on $\chi(M)$. Throughout this paper, the curvature tensor is defined by $R_{X Y} Z=\nabla_{X} \nabla_{Y} Z-\nabla_{Y} \nabla_{X} Z-\nabla_{[X, Y]} Z$ for all $X, Y, Z \in \chi(M)$ while the Riemann Christoffel curvature tensor is given by $R(X, Y, Z, W)=-g\left(R_{X Y} Z, W\right)$. The identity $(K 1)$ is often called Kaehler identity. It is known the fact that there exist non Kaehler manifolds satisfying $(K 1)$ identity (e.g. para-Kaehler manifolds, [11]).

\section{Warped product manifolds}

Singly warped products or simply warped products were first defined by Bishop and O'Neill in [1] in order to construct Riemannian manifolds with negative sectional curvature. Let $\left(B, g_{B}\right)$ and $\left(F, g_{F}\right)$ be Riemannian manifolds and let $b: B \longrightarrow(0, \infty)$ be a smooth function. The warped product $\widetilde{M}=B \times_{b} F$ is the product manifold $B \times F$ endowed with the metric $\widetilde{g}=g_{B} \oplus b^{2} g_{F}$. More precisely, if $\pi: B \times F \longrightarrow B$ and $\tau: B \times F \longrightarrow F$ are natural projections, the metric $g$ is defined by

$$
\widetilde{g}=\pi^{*} g_{B}+(b \circ \pi)^{2} \tau^{*} g_{F} .
$$

The function $b$ is called warping function. If $b \equiv 1$, then we have a product manifold.

If $X, Y$ are tangent to $B$ and $Z, W$ tangent to $F$, then the Levi-Civita connection $\widetilde{\nabla}$ of $\widetilde{M}$ is given by

$$
\left\{\begin{array}{l}
\widetilde{\nabla}_{X} Y=\nabla_{X}^{B} Y, \quad \widetilde{\nabla}_{X} Z=X(\ln b) Z \\
\widetilde{\nabla}_{Z} W=\nabla_{Z}^{F} W-b^{2} g_{F}(Z, W) \nabla^{B}(\ln b),
\end{array}\right.
$$

where $\nabla^{B}$ and $\nabla^{F}$ are the Levi-Civita connections on $B$, respectively on $F$, and $\nabla^{B}(\ln b)$ is the gradient of $\ln b$ with respect to the metric $g_{B}$. 
Let $(M, \varphi, \xi, \eta, g)$ be an almost contact metric manifold. Consider the warped product manifold $\widetilde{M}=\mathbb{R}_{+} \times_{t} M$, where $t>0$ is the global coordinate of $\mathbb{R}_{+}$, i.e., the metric $\widetilde{g}$ of $\widetilde{M}$ is defined by

$$
\widetilde{g}=d t^{2}+t^{2} g
$$

Let an endomorphism on $\chi(\widetilde{M})$ defined by

$$
J \partial_{t}=-\frac{1}{t} \xi, \quad J X=\varphi X+t \eta(X) \partial_{t}, \quad \forall X \in \chi(M),
$$

where $\partial_{t}=\frac{d}{d t}$. For $\widetilde{X}=(a, X) \in \chi(\widetilde{M}), a \in C^{\infty}\left(\mathbb{R}_{+}\right), X \in \chi(M)$ we have

$$
J \widetilde{X}=J(a, X)=\left(t \eta(X), \varphi X-\frac{a}{t} \xi\right) .
$$

The proofs of the following propositions are straightforward.

Proposition 2.1. $J$ is an almost complex structure compatible with the metric $\widetilde{g}$.

Proposition 2.2. The Levi-Civita connection $\widetilde{\nabla}$ of $\widetilde{g}$ is given by:

$$
\left\{\begin{array}{l}
\widetilde{\nabla}_{\partial_{t}} \partial_{t}=0, \quad \widetilde{\nabla}_{X} \partial_{t}=\widetilde{\nabla}_{\partial_{t}} X=\frac{1}{t} X \\
\widetilde{\nabla}_{X} Y=\nabla_{X} Y-t g(X, Y) \partial_{t}, \quad X, Y \in \chi(M) .
\end{array}\right.
$$

Proposition 2.3. The covariant derivative of $J$ is given by:

$$
\left\{\begin{array}{l}
\left(\widetilde{\nabla}_{\partial_{t}} J\right) \partial_{t}=(0,0), \quad\left(\widetilde{\nabla}_{\partial_{t}} J\right) X=(0,0) \\
\left(\widetilde{\nabla}_{X} J\right) \partial_{t}=\left(0,-\frac{1}{t}\left(\nabla_{X} \xi+\varphi X\right)\right) \\
\left(\widetilde{\nabla}_{X} J\right) Y=\left(t\left(\left(\nabla_{X} \eta\right)(Y)-g(X, \varphi Y)\right),\left(\nabla_{X} \varphi\right) Y-g(X, Y) \xi+\eta(Y) X\right) .
\end{array}\right.
$$

Corollary 2.4. $J$ is parallel if and only if

$$
\left\{\begin{array}{l}
\left(\widetilde{\nabla}_{X} \varphi\right) Y=g(X, Y) \xi-\eta(Y) X, \quad\left(\nabla_{X} \eta\right)(Y)=g(X, \varphi Y) \\
\nabla_{X} \xi=-\varphi X, \quad X, Y \in \chi(M)
\end{array}\right.
$$

i.e., $(\widetilde{M}, J, \widetilde{g})$ is Kaehler if and only if $(M, \varphi, \xi, \eta, g)$ is Sasakian.

Proposition 2.5. For the curvature of the manifold $\widetilde{M}$ we have

$$
\left\{\begin{array}{l}
\widetilde{R}\left(\partial_{t}, X\right) \partial_{t}=0, \widetilde{R}(X, Y) \partial_{t}=0, \widetilde{R}\left(\partial_{t}, X\right) Y=0 \\
\widetilde{R}(X, Y) Z=R(X, Y) Z-g(Y, Z) X+g(X, Z) Y
\end{array}\right.
$$

where $\widetilde{R}$ (respectively $R$ ) are the curvature tensors for $\widetilde{g}$ (respectively for $g$ ).

Moreover, the following relations hold:

$$
\left\{\begin{array}{l}
\widetilde{R}\left(\partial_{t}, X\right)\left(J \partial_{t}\right)=0, \widetilde{R}\left(\partial_{t}, X\right)(J Y)=0 \\
\widetilde{R}(X, Y)\left(J \partial_{t}\right)=-\frac{1}{t}[R(X, Y) \xi-\eta(Y) X+\eta(X) Y] \\
\widetilde{R}(X, Y)(J Z)=R(X, Y)(\varphi Z)-g(Y, \varphi Z) X+g(X, \varphi Z) Y
\end{array}\right.
$$


In the following we compute expressions of the form $\widetilde{g}(\widetilde{R}(A, B)(J C), J D)$. The useful following expressions are obtained in the cases:

1. $\widetilde{g}\left(\widetilde{R}(X, Y)\left(J \partial_{t}\right), J W\right)$ $=-t[g(R(X, Y) \xi, \varphi W)-\eta(Y) g(X, \varphi W)+\eta(X) g(Y, \varphi W)]$,

2. $\widetilde{g}\left(\widetilde{R}(X, Y)(J Z), J \partial_{t}\right)$ $=-t[\eta(R(X, Y)(\varphi Z))-\eta(X) g(Y, \varphi Z)+\eta(Y) g(X, \varphi Z)]$,

3. $\tilde{g}(\widetilde{R}(X, Y)(J Z), J W)$ $=t^{2}[g(R(X, Y) \varphi Z, \varphi W)-g(Y, \varphi Z) g(X, \varphi W)+g(X, \varphi Z) g(Y, \varphi W)]$.

Theorem 2.6. $\widetilde{M}$ is $(K 1)$ if and only if

$$
R(X, Y, Z, W)
$$

$$
\begin{aligned}
= & R(X, Y, \varphi Z, \varphi W)-g(X, \varphi Z) g(Y, \varphi W)+g(Y, \varphi Z) g(X, \varphi W) \\
& -g(Y, Z) g(X, W)+g(X, Z) g(Y, W) .
\end{aligned}
$$

Proof. $\widetilde{M}$ is $(K 1)$ if and only if $\widetilde{g}(\widetilde{R}(A, B)(J C), J D)=\widetilde{g}(\widetilde{R}(A, B) C, D)$ for all $A, B, C, D \in \chi(\widetilde{M})$. We have:

1.

$$
\begin{aligned}
& \widetilde{g}\left(\widetilde{R}(X, Y)\left(J \partial_{t}\right), J W\right)=\widetilde{g}\left(\widetilde{R}(X, Y) \partial_{t}, W\right) \\
\Longrightarrow & -t[g(R(X, Y) \xi, \varphi W)-\eta(Y) g(X, \varphi W)+\eta(X) g(Y, \varphi W)]=0 \\
\Longrightarrow & g(\varphi(R(X, Y) \xi-\eta(Y) X+\eta(X) Y), W)=0 \text { for every } W \\
\Longrightarrow & R(X, Y) \xi-\eta(Y) X+\eta(X) Y \in \operatorname{ker}(\varphi) .
\end{aligned}
$$

Thus,

$$
R(X, Y) \xi=\eta(Y) X-\eta(X) Y
$$

since both terms have no $\xi$-component.

2.

$$
\begin{aligned}
& \widetilde{g}\left(\widetilde{R}(X, Y)(J Z), J \partial_{t}\right)=\widetilde{g}\left(\widetilde{R}(X, Y) Z, \partial_{t}\right) \\
\Longrightarrow & \widetilde{g}\left(R(X, Y)(\varphi Z)-g(Y, \varphi Z) X+g(X, \varphi Z) Y,-\frac{1}{t} \xi\right) \\
= & \widetilde{g}\left(R(X, Y) Z-g(Y, Z) X+g(X, Z) Y, \partial_{t}\right) \\
\Longrightarrow & -\frac{1}{t} t^{2} \eta(R(X, Y)(\varphi Z)-g(Y, \varphi Z) X+g(X, \varphi Z) Y)=0 .
\end{aligned}
$$

Thus we have obtained,

$$
R(X, Y)(\varphi Z)-g(Y, \varphi Z) X+g(X, \varphi Z) Y \in \operatorname{ker}(\eta)
$$

3.

$$
\begin{aligned}
& \widetilde{g}(\widetilde{R}(X, Y)(J Z), J W)=\widetilde{g}(\widetilde{R}(X, Y) Z, W) \\
\Longrightarrow & \widetilde{g}\left(R(X, Y)(\varphi Z)-g(Y, \varphi Z) X+g(X, \varphi Z) Y, \varphi W+t \eta(W) \partial_{t}\right) \\
= & \widetilde{g}(R(X, Y) Z-g(Y, Z) X+g(X, Z) Y, W) .
\end{aligned}
$$


We obtain directly

$$
\begin{aligned}
& g(R(X, Y)(\varphi Z), \varphi W)-g(Y, \varphi Z) g(X, \varphi W)+g(X, \varphi Z) g(Y, \varphi W) \\
= & g(R(X, Y) Z, W)-g(Y, Z) g(X, W)+g(X, Z) g(Y, W)
\end{aligned}
$$

and consequently

$$
\begin{aligned}
& R(\varphi W, \varphi Z, X, Y)-R(W, Z, X, Y) \\
= & g(Y, \varphi Z) g(X, \varphi W)-g(X, \varphi Z) g(Y, \varphi W) \\
& +g(X, Z) g(Y, W)-g(Y, Z) g(X, W) .
\end{aligned}
$$

Note that (2) implies (3) and (4) implies (2).

As consequences we have

$$
\begin{aligned}
& R(\xi, Y, \xi, W)=g(Y, W) \\
& R(\xi, Y, Z, W)=R(\xi, Y, \varphi Z, \varphi W)=0, \\
& R(X, Y, Z, W)-g(Y, W) g(X, Z)+g(X, W) g(Y, Z) \\
= & R(X, Y, \varphi Z, \varphi W)-g(Y, \varphi W) g(X, \varphi Z)+g(X, \varphi W) g(Y, \varphi Z),
\end{aligned}
$$

where $X, Y, Z$ and $W$ are orthogonal to $\xi$.

Definition 2.7. We say that an almost contact metric manifold satisfies (G1)identity if its curvature tensor fulfills (1).

Proposition 2.8. The curvature tensor of a Sasakian manifold satisfies (G1) (see also Lemma 7.1 in [3]).

Proposition 2.9. Any contact manifold satisfying (G1) is Sasakian.

Proof. It is known (e.g. Proposition 7.6 from [3]) that a contact manifold is Sasakian if and only if $R(X, Y) \xi=\eta(Y) X-\eta(X) Y$ for all $X$ and $Y$.

Back to the cone manifold $\widetilde{M}$. We give:

Theorem 2.10. $\widetilde{M}$ is $(K 2)$ if and only if

$$
\begin{aligned}
& R(X, Y, Z, W) \\
= & R(\varphi X, Y, Z, \varphi W)+R(X, \varphi Y, Z, \varphi W)+R(X, Y, \varphi Z, \varphi W) \\
& +g(X, Z) \eta(W) \eta(Y)-g(Z, Y) \eta(X) \eta(W) .
\end{aligned}
$$

Proof. $\widetilde{M}$ is $(K 2)$ if and only if

$$
\widetilde{R}(A, B, C, D)=\widetilde{R}(J A, B, C, J D)+\widetilde{R}(A, J B, C, J D)+\widetilde{R}(A, B, J C, J D),
$$

where $A, B, C$ and $D$ are arbitrary vector fields on $\widetilde{M}$.

Three cases are essential:

1) $A=\partial_{t}, B=Y, C=\partial_{t}, D=W$ which is trivial.

2) $A=\partial_{t}, B=Y, C=Z, D=W$. 
One has:

$$
\begin{aligned}
\widetilde{R}\left(J \partial_{t}, Y, Z, J W\right) & =-\frac{1}{t} \widetilde{R}(\xi, Y, Z, \varphi W), \\
\widetilde{R}\left(\partial_{t}, J Y, Z, J W\right) & =0, \\
\widetilde{R}\left(\partial_{t}, Y, J Z, J W\right) & =0 .
\end{aligned}
$$

It follows that the right side is equal to:

$$
-\operatorname{tg}(\xi, R(Z, \varphi W) Y-g(\varphi W, Y) Z+g(Z, Y) \varphi W) .
$$

Since the left side vanishes, in this case we obtain

(6) $\quad R(\xi, Y, Z, \varphi W)=\eta(Z) g(\varphi W, Y)$ for every $Y, Z, W \in \chi(M)$.

3) $A=X, B=Y, C=Z, D=W$. One has

$$
\begin{aligned}
\widetilde{R}(J X, Y, Z, J W) & =\widetilde{R}(\varphi X, Y, Z, \varphi W), \\
\widetilde{R}(X, J Y, Z, J W) & =\widetilde{R}(X, \varphi Y, Z, \varphi W), \\
\widetilde{R}(X, Y, J Z, J W) & =\widetilde{R}(X, Y, \varphi Z, \varphi W) .
\end{aligned}
$$

It follows that the right side is equal to

$$
\begin{gathered}
t^{2}[R(\varphi X, Y, Z, \varphi W)+R(X, \varphi Y, Z, \varphi W)+R(X, Y, \varphi Z, \varphi W)]+ \\
+t^{2}[-g(\varphi W, \varphi Y) g(X, Z)+g(Z, Y) g(\varphi X, \varphi W)]
\end{gathered}
$$

while the left side is equal to:

$$
t^{2} R(X, Y, Z, W)+t^{2}[-g(W, Y) g(X, Z)+g(Z, Y) g(X, W)],
$$

and hence we have (5). Since (5) implies (6), we get the statement.

As consequences one has

$$
\begin{aligned}
& R(\xi, Y, \xi, W)=g(Y, W), \\
& R(\xi, Y, Z, W)=0, \\
& R(X, Y, Z, W)=R(\varphi X, Y, Z, \varphi W)+R(X, \varphi Y, Z, \varphi W)+R(X, Y, \varphi Z, \varphi W)
\end{aligned}
$$

for all $X, Y, Z, W$ orthogonal to $\xi$.

Definition 2.11. We say that an almost contact metric manifold satisfies (G2)-identity if its curvature tensor fulfills (5).

Let us focus our attention to the third identity of Gray.

Theorem 2.12. The manifold $\widetilde{M}$ is $(K 3)$ if and only if

$$
\begin{aligned}
& R(X, Y, Z, W) \\
= & R(\varphi X, \varphi Y, \varphi Z, \varphi W)+g(X, Z) \eta(W) \eta(Y)-g(Z, Y) \eta(X) \eta(W) \\
& +g(Y, W) \eta(X) \eta(Z)-g(X, W) \eta(Y) \eta(Z)
\end{aligned}
$$

for all $X, Y, Z, W \in \chi(M)$. 
Proof. $\widetilde{M}$ is $(K 3)$ if and only if $\widetilde{R}(A, B, C, D)=\widetilde{R}(J A, J B, J C, J D)$ for all $A, B, C, D \in \chi(\widetilde{M})$.

The essential cases are:

1) $A=\partial_{t}, B=Y, C=\partial_{t}, D=W$.

The left member vanishes and the right member is equal to $R(\xi, \varphi Y, \xi, \varphi W)-$ $g(\varphi W, \varphi Y)$. We get

$$
R(\xi, \varphi Y, \xi, \varphi W)=g(\varphi W, \varphi Y) .
$$

2) $A=\partial_{t}, B=Y, C=Z, D=W$.

The left hand vanishes and on the right hand we have $R(\xi, \varphi Y, \varphi Z, \varphi W)$. We get

$$
R(\xi, \varphi Y, \varphi Z, \varphi W)=0 .
$$

3) $A=X, B=Y, C=Z, D=W$.

Again, the left part is equal to

$$
t^{2}[R(X, Y, Z, W)-g(Z, X) g(W, Y)+g(Y, Z) g(X, W)]
$$

and the right part is equal to

$$
t^{2}[R(\varphi X, \varphi Y, \varphi Z, \varphi W)-g(\varphi W, \varphi Y) g(\varphi X, \varphi Z)+g(\varphi Y, \varphi Z) g(\varphi X, \varphi W)] .
$$

Hence (7) is proved. Note that (7) implies both (8) and (9).

Consequently

$$
\begin{aligned}
R(\xi, Y, \xi, W) & =g(Y, W), \\
R(\xi, Y, Z, W) & =0, \\
R(X, Y, Z, W) & =R(\varphi X, \varphi Y, \varphi Z, \varphi W)
\end{aligned}
$$

for all $X, Y, Z, W \in \chi(M)$ orthogonal to $\xi$.

Definition 2.13. We say that an almost contact metric manifold satisfies (G3)-identity if its curvature fulfills the relation (7).

\section{The Boothby-Wang fibration}

In this section we use the Boothby-Wang fibration [5] in order to strengthen previous results.

Let $M$ be a $(2 n+1)$-dimensional smooth manifold. A contact form on $M$ is a 1 -form $\eta$ satisfying

$$
\eta \wedge(d \eta)^{n} \neq 0
$$

We say that $\eta$ endows $M$ with a contact structure. It is clear that $\eta$ induces an orientation on $M$ and hence there is a global non vanishing vector field $\xi$ on $M$ so that $\eta(\xi)=1$. If $\xi$ is regular in the sense of Palais (see [10]), then the contact structure (and also $M$ ) is called regular. If moreover $M$ is compact, one can consider the space of all orbits of $\xi$, i.e., $N=M_{/ \xi}$ thus obtaining a smooth manifold. We have: 
Theorem A $([5])$. Let $(M, \eta)$ be a compact, regular, contact manifold. Then $M$ is a principal circle bundle over $N$ and $\eta$ is a connection form of this bundle. The curvature form $\Theta$ of $\eta$ defines a symplectic form on $N$.

This fibration $S^{1} \longrightarrow M \stackrel{\pi}{\longrightarrow} N$ is called the Boothby-Wang fibration.

Let $\Omega$ the symplectic 2 -form of $N$. We denote by $G$ the associated metric, i.e., $\Omega(X, Y)=G(X, J Y)$ with $J$ the almost complex structure.

In the following, by $X^{\uparrow}$ we denote the lift of a vector field $X \in \chi(N) . X^{\uparrow}$ is a horizontal vector field of $M$. On $M$ a $(1,1)$ tensor field $\varphi$ can be defined, namely

$$
\varphi X^{\uparrow}=(J X)^{\uparrow}, \quad \varphi \xi=0 .
$$

We can easily see that

$$
\varphi^{2}=-I+\eta \otimes \xi
$$

In this way, $(\varphi, \xi, \eta)$ becomes an almost contact structure. The metric $G$ can be lifted and hence one defines $g$ on $M$ as follows:

$$
g=\pi^{*} G+\eta \otimes \eta .
$$

The metric $g$ is compatible with the contact structure and $\xi=\eta^{\#}$.

Without loss of generality one can suppose $d \eta=\pi^{*} \Omega$ and thus we have

$$
g\left(X^{\uparrow}, \varphi Y^{\uparrow}\right)=G(X, J Y) \circ \pi=\Omega(X, Y) \circ \pi=\pi^{*} \Omega\left(X^{\uparrow}, Y^{\uparrow}\right)=d \eta\left(X^{\uparrow}, Y^{\uparrow}\right) .
$$

In this way, $(\varphi, \xi, \eta, g)$ becomes a contact metric structure on $M$.

If the symplectic structure of $N$ derives from a Kaehlerian structure $(J, G)$, the obtained structure on $M$ is Sasakian (i.e., contact and normal manifold). See e.g. [3]. But generally, a symplectic structure need not come from a Kaehlerian one. Yet, one can always find an almost Kaehlerian structure inducing it. In this case, the contact structure on the total space of a Boothby-Wang fibration is $K$-contact, i.e., the vector field $\xi$ is Killing, namely $\mathcal{L}_{\xi} g=0$. It easily follows that the integral curves of $\xi$ are geodesics.

It is easy to prove the relation

$$
\left[X^{\uparrow}, Y^{\uparrow}\right]=[X, Y]^{\uparrow}-2 G(X, J Y) \xi
$$

for all $X, Y \in \chi(N)$.

Denote by $\stackrel{M}{\nabla}$ and $\stackrel{N}{\nabla}$ the Levi-Civita connections on $M$ and $N$, respectively. We have

$$
g\left(\stackrel{M}{\nabla}_{X} Y^{\uparrow}, Z^{\uparrow}\right) \circ \pi=G(\stackrel{N}{\nabla} X Y, Z)
$$

for any $X, Y, Z \in \chi(N)$. For the vertical part we shall compute $\eta\left(\stackrel{M}{\nabla}_{X^{\uparrow}} Y^{\uparrow}\right)$ :

$$
\begin{aligned}
2 g\left(\nabla_{X^{\uparrow}}^{M} Y^{\uparrow}, \xi\right)= & X^{\uparrow} g\left(Y^{\uparrow}, \xi\right)+Y^{\uparrow} g\left(X^{\uparrow}, \xi\right)-\xi g\left(X^{\uparrow}, Y^{\uparrow}\right)+g\left(\left[X^{\uparrow}, Y^{\uparrow}\right], \xi\right) \\
& +g\left(\left[\xi, X^{\uparrow}\right], Y^{\uparrow}\right)+g\left(X^{\uparrow},\left[\xi, Y^{\uparrow}\right]\right) \\
= & \eta\left(\left[X^{\uparrow}, Y^{\uparrow}\right]\right)-\left(\mathcal{L}_{\xi} g\right)\left(X^{\uparrow}, Y^{\uparrow}\right) \\
= & -2 d \eta\left(X^{\uparrow}, Y^{\uparrow}\right) .
\end{aligned}
$$


We obtain

$$
\eta\left(\stackrel{M}{\nabla}_{X} Y^{\uparrow}\right) \circ \pi=-G(X, J Y) .
$$

In the following, we will ignore $\pi$, due to the isomorphism between the horizontal distribution of $T(M)$ and $T(N)$. Hence

$$
\stackrel{M}{\nabla}_{X \uparrow}^{\uparrow} Y^{\uparrow}=\left(\stackrel{N}{\nabla}_{X} Y\right)^{\uparrow}-G(X, J Y) \xi
$$

In the same way, one can show

$$
\stackrel{M}{\nabla}_{X \uparrow} \xi=-\varphi X^{\uparrow} .
$$

Denote by $R^{M}$ and $R^{N}$ the curvature tensors of $M$ and $N$, respectively. Then

$$
\begin{aligned}
& R^{M}\left(X^{\uparrow}, Y^{\uparrow}\right) Z^{\uparrow} \\
= & \left(R^{N}(X, Y) Z\right)^{\uparrow}+g\left(Y^{\uparrow}, \varphi Z^{\uparrow}\right) \varphi X^{\uparrow}-g\left(X^{\uparrow}, \varphi Z^{\uparrow}\right) \varphi Y^{\uparrow}-2 g\left(x^{\uparrow}, \varphi Y^{\uparrow}\right) \varphi Z^{\uparrow} \\
& +\left\{g\left(X^{\uparrow},\left(\stackrel{M}{\nabla} Y^{\uparrow} \varphi\right) Z^{\uparrow}\right)-g\left(Y^{\uparrow},\left(\stackrel{M}{\nabla}_{X^{\uparrow}} \varphi\right) Z^{\uparrow}\right)\right\} \xi
\end{aligned}
$$

and hence

$$
\begin{aligned}
R^{M}\left(W^{\uparrow}, Z^{\uparrow}, X^{\uparrow}, Y^{\uparrow}\right)= & R^{N}(W, Z, X, Y) \circ \pi-2 g\left(X^{\uparrow}, \varphi Y^{\uparrow}\right) g\left(W^{\uparrow}, \varphi Z^{\uparrow}\right) \\
& +g\left(Y^{\uparrow}, \varphi Z^{\uparrow}\right) g\left(W^{\uparrow}, \varphi X^{\uparrow}\right)-g\left(X^{\uparrow}, \varphi Z^{\uparrow}\right) g\left(W^{\uparrow}, \varphi Y^{\uparrow}\right) .
\end{aligned}
$$

Suppose that the base manifold $N$ satisfies Gray identities. What are the corresponding curvature identities for the upstairs manifold $M$ ?

If $N$ is $\left(K_{1}\right)$, then

$$
\begin{aligned}
& R^{M}\left(X^{\uparrow}, Y^{\uparrow}, \varphi Z^{\uparrow}, \varphi W^{\uparrow}\right)-R^{M}\left(X^{\uparrow}, Y^{\uparrow}, Z^{\uparrow}, W^{\uparrow}\right) \\
= & -g\left(Y^{\uparrow}, W^{\uparrow}\right) g\left(Z^{\uparrow}, X^{\uparrow}\right)-g\left(Y^{\uparrow}, \varphi W^{\uparrow}\right) g\left(Z^{\uparrow}, \varphi X^{\uparrow}\right) \\
& +g\left(X^{\uparrow}, W^{\uparrow}\right) g\left(Z^{\uparrow}, Y^{\uparrow}\right)+g\left(X^{\uparrow}, \varphi W^{\uparrow}\right) g\left(Z^{\uparrow}, \varphi Y^{\uparrow}\right) .
\end{aligned}
$$

If $N$ is $\left(K_{2}\right)$, then

$$
\begin{aligned}
& R^{M}\left(\varphi X^{\uparrow}, Y^{\uparrow}, Z^{\uparrow}, W^{\uparrow}\right)+R^{M}\left(X^{\uparrow}, \varphi Y^{\uparrow}, Z^{\uparrow}, W^{\uparrow}\right) \\
& +R^{M}\left(X^{\uparrow}, Y^{\uparrow}, \varphi Z^{\uparrow}, W^{\uparrow}\right)+R^{M}\left(X^{\uparrow}, Y^{\uparrow}, Z^{\uparrow}, \varphi W^{\uparrow}\right)=0 .
\end{aligned}
$$

If $N$ is $\left(K_{3}\right)$, then

$$
R^{M}\left(\varphi X^{\uparrow}, \varphi Y^{\uparrow}, \varphi Z^{\uparrow}, \varphi W^{\uparrow}\right)-R^{M}\left(X^{\uparrow}, Y^{\uparrow}, Z^{\uparrow}, W^{\uparrow}\right)=0 .
$$

These relations are exactly the defined Gray identities for almost contact metric manifolds for vector fields orthogonal to $\xi$. 


\section{Properties and examples}

In their paper [9], D. Janssens and L. Vanhecke have studied curvature tensors for almost contact metric structures and defined almost $C(\alpha)$-manifolds, namely those almost contact metric manifolds whose curvature tensor satisfies the following property:

$$
\begin{aligned}
& \exists \alpha \in \mathbb{R} \text { such that for all } X, Y, Z, W \in \chi(M) \\
& R(X, Y, Z, W)= R(X, Y, \varphi Z, \varphi W)+\alpha\{g(X, Z) g(Y, W)-g(X, W) g(Y, Z) \\
&-g(X, \varphi Z) g(Y, \varphi W)+g(X, \varphi W) g(Y, \varphi Z)\} .
\end{aligned}
$$

(In the original paper [9] different signs appear due the fact that the Riemann Christoffel curvature tensor is defined with the opposite sign.) This means that manifolds satisfying the first Gray identity $\left(K_{1 \varphi}\right)$ in the sense of Bonome et al. are in fact $C(0)$-manifolds, while that manifolds satisfying $(G 1)$ are $C(1)$-manifolds. Note that cosymplectic, Sasakian and Kenmotsu manifolds are respectively $C(0), C(1)$ and $C(-1)$ manifolds (see Theorem 2.3 , in [9]).

Let us come back to Gray identities for an almost Hermitian manifold.

It is known that $K 1 \Rightarrow K 2 \Rightarrow K 3$ (see [8], §5). Consequently we have:

Proposition 4.1. For a class $\mathcal{L}$ of almost contact metric manifolds, denote by $\mathcal{L}_{i}$ the subclass of manifolds whose curvature satisfies $G i, i=1,2,3$. Then we have the following inclusions

$$
\mathcal{L}_{1} \subseteq \mathcal{L}_{2} \subseteq \mathcal{L}_{3} \subseteq \mathcal{L}
$$

As Gray remarked for Kaehlerian manifolds, we can say that as $i$ decreases, a manifold in $\mathcal{L}_{i}$ resembles Sasakian manifold more closely.

Proposition 4.2. Let $(M, \varphi, \xi, \eta, g)$ be a K-contact manifold satisfying $G 1$ curvature identity. Then the manifold $M$ is Sasakian.

Proof. By using Proposition 7.5 in [3, p. 94], a K-contact manifold whose curvature satisfies $R_{X Y} \xi=\eta(Y) X-\eta(X) Y$ is Sasakian. But this last relation is a consequence of G1 identity. See also Proposition 2.9.

Proposition 4.3. Let $M$ be a contact metric manifold for which $\xi$ belongs to the $(\kappa, \mu)$-nullity distribution, namely its curvature satisfies

$$
R_{X Y} \xi=\kappa(\eta(Y) X-\eta(X) Y)+\mu(\eta(Y) h X-\eta(X) h Y),
$$

where $h=\frac{1}{2} \mathcal{L}_{\xi} \varphi$ and $\kappa, \mu$ are constants. Suppose $M$ satisfies (G1) identity. Then $M$ is Sasakian.

Proof. If $M$ is $(G 1)$, then $R_{X Y} \xi=\eta(Y) X-\eta(X) Y$ for all $X, Y \in \chi(M)$. Combining with the fact that $\xi$ belongs to the $(\kappa, \mu)$-nullity distribution we obtain

$$
(\kappa-1)(\eta(Y) X-\eta(X) Y)+\mu(\eta(Y) h X-\eta(X) h Y)=0
$$


for all $X, Y \in \chi(M)$. If $\mu \neq 0$ this implies $h Y=\frac{1-\kappa}{\mu} Y$ for all $Y \in \operatorname{ker}(\eta)$. We know that $h$ anticommutes with $\varphi$ and hence one gets $\kappa=1$. But using $[3$, Theorem 7.7 , p. 103], it follows that $M$ is a Sasakian manifold. If $\mu=0$ we immediately have $\kappa=1$.

Proposition 4.4. Let $(M, \varphi, \eta, \xi, g)$ be a contact metric manifold satisfying (G3) identity. Then $M$ is K-contact.

Proof. Choose a $\varphi$-adapted local orthonormal frame on $M$, namely $\left\{X_{i}, \varphi X_{i}\right.$, $\xi\}, i=1, \ldots, n$. Since $M$ is $(G 3)$ the relation $R(X, \xi, Y, \xi)=g(X, Y)$ holds for all $X, Y \in \operatorname{ker}(\eta)$. Taking $X=Y=X_{i}$ (respectively $X=Y=\varphi X_{i}$ ) one immediately obtains $\operatorname{Ric}(\xi, \xi)=2 n$, where $\operatorname{Ric}$ is the Ricci tensor on $M$. Now we use the fact that a contact metric manifold is K-contact if and only if the Ricci tensor in the direction of the characteristic vector field $\xi$ is equal to $2 n$ ([2, Theorem, p. 65]).

As consequence, we can state:

Proposition 4.5. If $(M, \varphi, \eta, \xi, g)$ is a contact metric manifold satisfying $(G 1)$ identity, then it is Sasakian.

Proof. The statement follows from Propositions 4.2 and 4.4.

\subsection{The generalized Heisenberg group $H(p, 1)$}

It is defined as the set of matrices of real numbers having the form

$$
a=\left[\begin{array}{rrr}
1 & A & c \\
0 & I_{p} & { }^{t} B \\
0 & 0 & 1
\end{array}\right],
$$

where $I_{p}$ is the identity $p \times p$ matrix, $A=\left(a_{1}, \ldots, a_{p}\right), B=\left(b_{1}, \ldots, b_{p}\right) \in \mathbb{R}^{p}$ and $c \in \mathbb{R}$. (Cf. [7].) $H(p, 1)$ is a connected, simply connected nilpotent Lie group of dimension $2 p+1$. We will consider $p=2$. A global system of coordinates $\left(x^{1}, x^{2}, y^{1}, y^{2}, z\right)$ on $H(2,1)$ is defined by $x^{i}(a)=a_{i}, y^{i}(a)=b_{i}$ for $i=1,2$ and $z(a)=c$. The global vector fields

$$
X_{i}=2 \frac{\partial}{\partial x^{i}}, Y_{i}=2\left(\frac{\partial}{\partial y^{i}}+x^{i} \frac{\partial}{\partial z}\right) \text { for } i=1,2, \text { and } \xi=2 \frac{\partial}{\partial z}
$$

are left invariant. We consider $\eta=\frac{1}{2}\left(d z-x^{1} d y^{1}-x^{2} d y^{2}\right)$ and the metric

$$
g=\frac{1}{4}\left(d x^{1} \otimes d x^{1}+d x^{2} \otimes d x^{2}+d y^{1} \otimes d y^{1}+d y^{2} \otimes d y^{2}\right)+\eta \otimes \eta .
$$

By direct computations we obtain that $d \eta=-\frac{1}{2}\left(d x^{1} \wedge d y^{1}+d x^{2} \wedge d y^{2}\right)$ and $\xi$ is the characteristic vector field, namely $\eta(\xi)=1$ and $i_{\xi} d \eta=0$. Moreover, the basis defined above is orthonormal: $g\left(X_{i}, X_{j}\right)=g\left(Y_{i}, Y_{j}\right)=\delta_{i j}, g(\xi, \xi)=1$ and $g\left(X_{i}, Y_{j}\right)=g\left(X_{i}, \xi\right)=g\left(Y_{i}, \xi\right)=0$. One has $\left[X_{i}, Y_{i}\right]=2 \xi$ for $i=1,2$ 
and the other brackets are equal to zero. Therefore it is easy to verify that the Levi-Civita connection is given by the following formulas:

$$
\begin{gathered}
\nabla_{\xi} X_{i}=-Y_{i}=\nabla_{X_{i}} \xi, \\
\nabla_{\xi} Y_{i}=X_{i}=\nabla_{Y_{i}} \xi, \\
\nabla_{X_{i}} Y_{i}=-\nabla_{Y_{i}} X_{i}=\xi
\end{gathered}
$$

for $i=1,2$, the other derivatives being zero. We compute also the RiemannChristoffel curvature tensor field:

$$
\begin{gathered}
R\left(X_{1}, X_{2}, Y_{1}, Y_{2}\right)=-1, \quad R\left(X_{1}, Y_{2}, X_{2}, Y_{1}\right)=-1, \\
R\left(X_{1}, Y_{1}, X_{2}, Y_{2}\right)=-2, \quad R\left(X_{i}, Y_{i}, X_{i}, Y_{i}\right)=-3, \\
R\left(X_{i}, \xi, X_{i}, \xi\right)=1, \quad R\left(Y_{i}, \xi, Y_{i}, \xi\right)=1 \quad \text { for } i=1,2 .
\end{gathered}
$$

The other values are zero or can be obtained from these ones.

Define $\varphi$ by:

$$
\varphi X_{1}=\cos \theta Y_{1}+\sin \theta Y_{2}, \varphi X_{2}=\varepsilon \sin \theta Y_{1}-\varepsilon \cos \theta Y_{2},
$$

$\varphi Y_{1}=-\cos \theta X_{1}-\varepsilon \sin \theta X_{2}, \varphi Y_{2}=-\sin \theta X_{1}+\varepsilon \cos \theta X_{2}, \varphi \xi=0, \varepsilon= \pm 1$.

Hence $(H(2,1), \varphi, \xi, \eta, g)$ is an almost contact metric manifold.

Proposition 4.6. The structure is quasi Sasakian. Moreover, it is K-contact if and only if $\varepsilon=-1$ and $\theta=0$. In this case $H(2,1)$ becomes a Sasakian manifold.

Proof. It can be proved that $\nabla_{\xi} \varphi=0$. Then for every $X, Y \in \chi(M)$ we have

$$
g\left(\nabla_{X} \xi, Y\right)+g\left(\nabla_{Y} \xi, X\right)=0,
$$

which means that $\xi$ is Killing. The following relation holds on $H(2,1)$ :

$$
g\left(Y,\left(\nabla_{X} \varphi\right) Z\right)=\eta(Y)\left(\nabla_{\varphi X} \eta\right)(Z)+\eta(Z)\left(\nabla_{Y} \eta\right)(\varphi X)
$$

which characterizes quasi Sasakian manifolds. Direct computations yield the second part of the statement.

Moreover, we can give the following:

Proposition 4.7. On $H(2,1)$

(1) the G3 identity holds if and only if $\cos \theta=0$ or $\sin \theta=0$ or $\varepsilon=1$;

(2) the $G 2$ identity holds if and only if $\varepsilon=1$ or $\sin \theta=0$;

(3) the $G 1$ identity holds if and only if $\varepsilon=-1$ and $\sin \theta=0$.

Proof. Straightforward computations. 


\subsection{Other examples}

Let $(N, \bar{g}, J)$ be an almost Hermitian manifold. Consider the warped product manifold $M=\mathbb{R} \times_{f} N$, where $f=f(\theta)$ is the warping function and $\theta$ is the global parameter on $\mathbb{R}$. Denote by $g=d \theta^{2}+f^{2}(\theta) \bar{g}$ the Riemannian metric on $M$. Define the global vector field $\xi=\frac{\partial}{\partial \theta}$ and the 1 -form $\eta=d \theta$. Define also the $(1,1)$ tensor field $\varphi$ by $\varphi X=J X$ if $X$ is tangent to $N$ and $\varphi \frac{\partial}{\partial \theta}=0$. Thus $(\varphi, \xi, \eta, g)$ is an almost contact metric structure on $M$. If $\bar{\nabla}$ and $\nabla$ are the Levi-Civita connections on $N$, respectively on $M$, we have

$$
\nabla_{\xi} X=\nabla_{X} \xi=\frac{f^{\prime}}{f} X, \quad \nabla_{\xi} \xi=0, \quad \nabla_{X} Y=\bar{\nabla}_{X} Y-f f^{\prime} \bar{g}(X, Y) \xi
$$

for all $X, Y$ tangent to $N$.

The Riemann Christoffel curvature tensor is given by

$$
\begin{aligned}
& R(W, \xi, X, Y)=0, \quad R(W, \xi, X, \xi)=-\frac{f^{\prime \prime}}{f} g(X, W), \\
& R(W, Z, X, Y)=f^{2}\left[\bar{R}(W, Z, X, Y)+\left(f^{\prime}\right)^{2}(\bar{g}(X, Z) \bar{g}(Y, W)-\bar{g}(Y, Z) \bar{g}(X, W))\right] .
\end{aligned}
$$

In order to have one of the three curvature identities we directly have

$$
\frac{f^{\prime \prime}}{f}=-1
$$

which implies that $f=\alpha \cos \theta+\beta \sin \theta$ with $\alpha$ and $\beta$ real constants. Now one can state the following:

Proposition 4.8. The manifold $M$ is $G 2$ (respectively G3) if and only if the almost Hermitian manifold $N$ is K2 (respectively K3).

Proof. One has the following relations:

$$
\begin{aligned}
& R(\varphi W, Z, X, \varphi Y)+R(W, \varphi Z, X, \varphi Y)+R(W, Z, \varphi X, \varphi Y) \\
= & f^{2}[\bar{R}(J W, Z, X, J Y)+\bar{R}(W, J Z, X, J Y)+\bar{R}(W, Z, J X, J Y)] \\
& +\left(f^{\prime}\right)^{2} f^{2}(\bar{g}(X, Z) \bar{g}(Y, W)-\bar{g}(Y, Z) \bar{g}(X, W))
\end{aligned}
$$

and

$R(W, Z, X, Y)-R(\varphi W, \varphi Z, \varphi X, \varphi Y)=f^{2}[\bar{R}(W, Z, X, Y)-\bar{R}(J W, J Z, J X, J Y)]$.

Hence the statement.

Remark 4.9. If $\operatorname{dim} N \geq 4$, then the manifold $M$ cannot be $G 1$.

Proof. Suppose $M$ satisfies $G 1$ identity. A straightforward computation gives

$$
\begin{aligned}
& \bar{R}(W, Z, J X, J Y)-\bar{R}(W, Z, X, Y) \\
= & \left(1+\left(f^{\prime}\right)^{2}\right)[\bar{g}(J X, W) \bar{g}(J Y, Z)-\bar{g}(J X, Z) \bar{g}(J Y, W) \\
& +\bar{g}(Y, W) \bar{g}(X, Z)-\bar{g}(Y, Z) \bar{g}(X, W)] .
\end{aligned}
$$


Since $f$ depends on $\theta$ (and it is not linear) while $\bar{g}$ and $\bar{R}$ do not, it follows that $N$ is $K 1$ and

$\bar{g}(J X, W) \bar{g}(J Y, Z)-\bar{g}(J X, Z) \bar{g}(J Y, W)+\bar{g}(Y, W) \bar{g}(X, Z)-\bar{g}(Y, Z) \bar{g}(X, W)=0$

for all $X, Y, Z, W$ tangent to $N$. This yields

$$
\bar{g}(J Y, Z) J X-\bar{g}(J X, Z) J Y+\bar{g}(X, Z) Y-\bar{g}(Y, Z) X=0 .
$$

If $\operatorname{dim} N \geq 4$ we can choose $X$ and $Y$ so that $X, Y, J X$ and $J Y$ are linearly independent, so, the previous equality is impossible.

Example 4.10. On $\mathbb{R}^{4}$ consider the global coordinates $x, y, u$ and $v$ respectively. Let $z \in I=(0, \pi / 2)$. Define the warped product $M=I \times{ }_{f} \mathbb{R}^{4}$, where the warping function $f: I \longrightarrow \mathbb{R}$ is given by $f(z)=\cos z$. More precisely, consider the Riemannian metric $g=d z^{2}+\cos ^{2} z\left(d x^{2}+d y^{2}+d u^{2}+d v^{2}\right)$. Let us define the almost contact structure by: $\xi=\partial_{z}, \eta=d z, \varphi \partial_{x}=\partial_{y}, \varphi \partial_{y}=-\partial_{x}$, $\varphi \partial_{u}=\partial_{v}, \varphi \partial_{v}=-\partial_{u}$ and $\varphi \partial_{z}=0$. Then $M$ is $G 2$ but not $G 1$.

The same result holds if on $M$ consider the warped metric

$$
g=d z^{2}+\sin ^{2} z\left(d x^{2}+d y^{2}+d u^{2}+d v^{2}\right) .
$$

This kind of structure is called sine-cone and gives way to construct many geometric objects (e.g. nearly Kaehler structures starting from a 5-dimensional Sasaki Einstein manifold). Cf. [6].

Proposition 4.11. Let $N$ be a surface and consider the warped product manifold $M=I \times N$, where $I$ is an open interval. Then $M$ satisfies $G 1$.

Proof. Being a surface, $N$ is automatically Kaehler. The almost contact structure is defined as in the beginning of this section. The statement follows from the fact that a Kaehler manifold is $K 1$ and the equation (10) is satisfied in dimension 2 .

\subsection{Hypersurfaces of almost Hermitian manifolds}

Let $(\widetilde{M}, J, \widetilde{g})$ a $(2 n+2)$-dimensional Kaehler manifold, and let $M$ be a totally umbilical (real) hypersurface in $\widetilde{M}$. Denote by $N$ the unit normal on $M$ and let $A, h$ be the Weingarten operator and the scalar-valued second fundamental form, respectively. As $M$ is totally umbilical, we have that $A X=\beta X$ for all $X$ tangent to $M$, with $\beta \in C^{\infty}(M)$.

It is well known the fact that on $M$ we can define an almost contact metric structure (see e.g. [3]). More precisely, we take $\xi=-J N$ and for $X \in \chi(M)$ we decompose $J X$ as:

$$
J X=\varphi X+\eta(X) N .
$$

Let $g$ be the restriction of the metric $\widetilde{g}$ on $M$. Denote by $\widetilde{\nabla}$ (respectively $\nabla$ ) the Levi-Civita connection on $\widetilde{M}$ (respectively on $M$ ). Then, by the formula of Gauss, one has

$$
\widetilde{\nabla}_{X} \xi=\nabla_{X} \xi+h(X, \xi) N .
$$


On the other hand, we have $\widetilde{\nabla}_{X} \xi=-J \widetilde{\nabla}_{X} N=J A X=\varphi A X+\eta(A X) N$. Hence

$$
\nabla_{X} \xi=\varphi A X \quad \text { and } \quad h(X, \xi)=\eta(A X) .
$$

Suppose now that $M$ satisfies the (G3) identity. This implies

$$
R(X, \xi, Y, \xi)=g(X, Y) \quad \forall X, Y \in \operatorname{ker}(\eta) .
$$

We should compute $R(X, \xi) \xi=\nabla_{X} \nabla_{\xi} \xi-\nabla_{\xi} \nabla_{X} \xi-\nabla_{[X, \xi]} \xi$. Since $M$ is totally umbilical, we have $\nabla_{X} \xi=\beta \varphi X$. Thus $\nabla_{\xi} \xi=0$. Then

$$
\nabla_{\xi} \nabla_{X} \xi=\xi(\beta) \varphi X+\beta\left(\nabla_{\xi} \varphi\right) X+\beta \varphi \nabla_{\xi} X
$$

But

and so

$$
\nabla_{\xi} X=\beta \varphi X-[X, \xi]
$$

It follows that

$$
\nabla_{\xi} \nabla_{X} \xi=\xi(\beta) \varphi X+\beta\left(\nabla_{\xi} \varphi\right) X+\beta^{2} \varphi^{2} X-\beta \varphi[X, \xi]
$$

$$
R(X, \xi) \xi=-\xi(\beta) \varphi X-\beta\left(\nabla_{\xi} \varphi\right) X+\beta^{2} X .
$$

Now, due to the fact $M$ is Kaehler, we have $\widetilde{\nabla}(J Y)=J \widetilde{\nabla}_{X} Y=J\left(\nabla_{X} Y+h(X, Y) N\right)=\varphi \nabla_{X} Y+\eta\left(\nabla_{X} Y\right) N-h(X, Y) \xi$.

On the other hand

$$
\widetilde{\nabla}(J Y)=\widetilde{\nabla}_{X}(\varphi Y+\eta(Y) N)=\nabla(\varphi Y)+h(X, \varphi Y) N+X \eta(Y) N-\eta(Y) \beta X .
$$

Identifying the tangent and the normal parts of $\widetilde{\nabla}(J Y)$ we obtain

$$
\begin{gathered}
\left(\nabla_{X} \varphi\right) Y=\beta \eta(Y) X-\beta g(X, Y) \xi, \\
\left(\nabla_{X} \eta\right)(Y)=-\beta g(X, \varphi Y),
\end{gathered}
$$

respectively.

Putting $X=\xi$ in $(12)$ we have $\left(\nabla_{\xi} \varphi\right) Y=\beta \eta(Y) \xi-\beta g(\xi, Y) \xi=0$ which implies

$$
\nabla_{\xi} \varphi=0
$$

Then

From (11) we have

$$
R(X, \xi) \xi=-\xi(\beta) \varphi X+\beta^{2} X
$$

$$
g\left(\beta^{2} X-X-\xi(\beta) \varphi X, Y\right)=0, \quad \forall Y \in \operatorname{ker}(\eta) .
$$

As $X$ and $\varphi X$ are linearly independent (and belong to $\operatorname{ker}(\eta)$ ), we obtain $\beta= \pm 1$.

Consequently

$$
A X= \pm X \quad \text { and } \quad h(X, Y)= \pm g(X, Y)
$$

For $\beta=-1$ it follows that

$$
\left(\nabla_{X} \varphi\right) Y=g(X, Y) \xi-\eta(Y) X .
$$

According to Theorem 6.14 in [3] this implies that $M$ is Sasakian. 
Proposition 4.12. Let $M$ be a totally umbilical hypersurface of a Kaehler manifold $\widetilde{M}$ endowed with the usual almost contact metric structure. If $M$ satisfies $G 3$ identity, then $M$ is a Sasakian manifold and hence $M$ satisfies all Gi for $i=1,2,3$.

More generally, if the second fundamental form of $M$ is given by

$$
h(X, Y)=\lambda \eta(X) \eta(Y)+\mu g(X, Y), \quad \forall X, Y \in \chi(M)
$$

with $\lambda$ and $\mu$ smooth functions on $M$, i.e., $M$ is totally quasi umbilical, and if $M$ satisfies (G3) identity, then it is Sasakian. As consequence, there is no cylindrical submanifold satisfying $(G 3)$ and whose second fundamental form is $h(X, Y)=\lambda \eta(X) \eta(Y)$.

Acknowledgements. The authors would like to thank Professor S. Ianus for suggesting this subject and for constant support during the preparation of this paper. They also wish to express their gratitude to Professors L. Ornea and D. E. Blair for their useful comments and critical remarks on the first version of the manuscript.

The authors are grateful to the anonymous referee for his or her valuable comments on this paper.

\section{References}

[1] R. L. Bishop and B. O'Neill, Manifolds of negative curvature, Trans. Amer. Math. Soc. 145 (1969), 1-49.

[2] D. E. Blair, Contact Manifolds in Riemannian Geometry, Springer-Verlag, Berlin-New York, 1976.

[3] _ Riemannian geometry of Contact and Symplectic Manifolds, Progess in Mathematics, Birkhäuser Boston, 2002.

[4] A. Bonome, L. M. Hervella, and I. Rozas, On the classes of almost Hermitian structures on the tangent bundle of an almost contact metric manifold, Acta Math. Hungar. 56 (1990), no. 1-2, 29-37.

[5] W. M. Boothby and H. C. Wang, On contact manifolds, Ann. of Math. (2) 68 (1958), $721-734$.

[6] M. Fernández, S. Ivanov, V. Muñoz, and L. Ugarte, Nearly hypo structures and compact nearely Kähler 6-manifolds with conical singularities, J. London Math. Soc. 78 (2008), no. 3, 580-604.

[7] J. C. Gonzalez and D. Chinea, Quasi-Sasakian homogeneous structures on the generalized Heisenberg group $H(p, 1)$, Proc. Amer. Math. Soc. 105 (1989), no. 1, 173-184.

[8] A. Gray, Curvature identities for Hermitian and almost Hermitian manifolds, Tôhoku Math. J. (2) 28 (1976), no. 4, 601-612.

[9] D. Janssens and L. Vanhecke, Almost contact structures and curvature tensors, Kodai Math. J. 4 (1981), no. 1, 1-27.

[10] R. S. Palais, A global formulation of the Lie theory of transformation groups, Mem. Amer. Math. Soc. No. 22 (1957), iii+123 pp.

[11] G. B. Rizza, Varietà parakähleriane, Ann. Mat. Pura Appl. (4) 98 (1974), 47-61.

[12] F. Tricerri and L. Vanhecke, Curvature tensors on almost Hermitian manifolds, Trans. Amer. Math. Soc. 267 (1981), no. 2, 365-397.

[13] L. Vanhecke, Almost Hermitian manifolds with J-invariant Riemann curvature tensor, Rend. Sem. Mat. Univ. Politec. Torino 34 (1975/76), 487-498. 
RALUCA MOCANU

FACUlty of Mathematics

UNIVERSITY OF BUCHAREST

Str. ACADEMiei N.14

s 1, Bucharest, Romania

E-mail address: xipita@yahoo.com

Marian IOAN MUNTEANU

FACULTy OF MATHEMATICS

UNIVERSITY 'Al.I.CUZA' OF IAŞI

Bd. Carol I, NO.11

700506 IAŞI, ROMANIA

E-mail address: marian.ioan.munteanu@gmail.com 